\title{
Numerical modeling of the contact between three-dimensional regions with the use of FEM
}

\author{
Tomasz Skrzypczak ${ }^{1, *}$, Ewa Węgrzyn-Skrzypczak ${ }^{2}$, Leszek Sowa ${ }^{1}$ \\ ${ }^{1}$ Czestochowa University of Technology, Faculty of Mechanical Engineering and Computer Science, \\ Institute of Mechanics and Machine Design Fundamentals, Dąbrowskiego 73, 42-201 Częstochowa, \\ Poland \\ ${ }^{2}$ Czestochowa University of Technology, Faculty of Mechanical Engineering and Computer Science, \\ Institute of Mathematics, Armii Krajowej 21, 42-201 Częstochowa, Poland
}

\begin{abstract}
The numerical approach based on the finite element method (FEM) for modeling of mechanical interaction between three-dimensional objects is presented in the paper. The model of contact is based on the assumption that the nodes of the region which is the source of contact cannot overlap with the nodes of the region being the target. The procedure of the detection of collision between surfaces of the source and the target is discussed in details. The behaviour of surfaces being in contact depends on their rigidity and is numerically modeled in the case of perfectly rigid source and deformable target. Each modeled object has an independent mesh of finite elements. These meshes can be freely moved relative to each other. Example of calculation using original program written in $\mathrm{C}++$ is presented and discussed.
\end{abstract}

Keywords: computational mechanics, contact problem, finite element method, multi-mesh approach

\section{Introduction}

Mechanical contact between working elements is very common in engineering practice. Various kinds of machine parts work together going into the mechanical and also thermal interactions. Mechanical contact is the result of direct impacts of their external boundaries. In general such interactions occur between the deformable bodies although often for the sake of simplicity is assumed perfect rigidity of one or more bodies.

The first work focused on the classical contact mechanics was written by Heinrich Hertz in 1881 [1]. Hertz investigated the contact problem between two elastic bodies with curved surfaces. Nearly one hundred years later Johnson, Kendall, and Roberts found a similar solution for the contact with adhesion [2]. Their model of contact is known as JKR model of elastic contact. Different approach called DMT model was proposed by Derjaguin and co-workers in 1975 [3]. Bowden and Tabor investigated the importance of surface roughness in contact process [4]. Archard in 1957 was the first who found that the area of contact is approximately proportional to the normal force [5]. Nowadays advanced numerical methods such as finite element method (FEM) and the development of computer

\footnotetext{
* Corresponding author: t.skrzypczak@imipkm.pcz.pl

Reviewers: Marek Macko, Branislav Ftorek
} 
technology makes possible numerical modeling of complex problems [6-8]. This work aims to present a method of detecting collisions between objects surfaces and matching them during elastic deformation.

\section{Mathematical model}

Two elastic objects are considered (Fig. 1). The first one called the target is fixed and not deformable, while the second called the source is mobile and deformable. In the initial phase, the objects are separated, then the movement of the source causes contact and deformation.

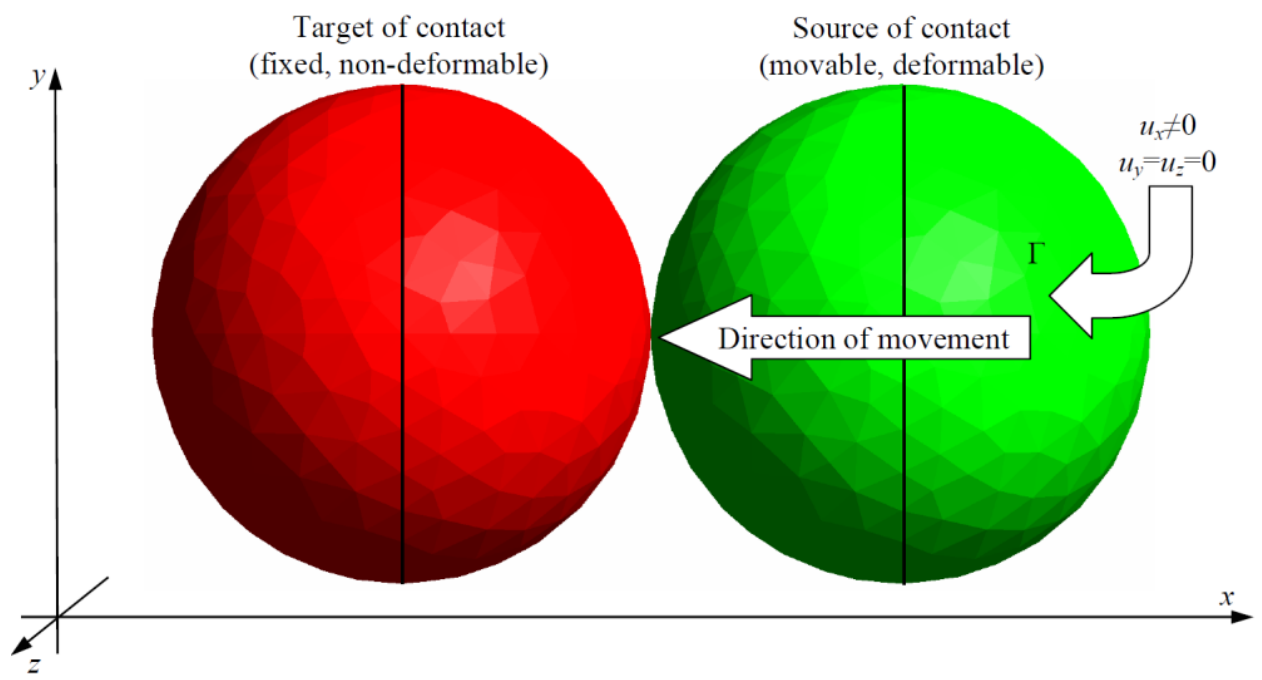

Fig. 1. Objects in contact

Mathematical model of deformations during the mechanical contact is based on the equilibrium equations of linear elasticity. Because the target of the contact is nondeformable and fixed below equations is only written for the source of the contact:

$$
\begin{aligned}
& \frac{\partial \sigma_{x}}{\partial x}+\frac{\partial \tau_{x y}}{\partial y}+\frac{\partial \tau_{x z}}{\partial z}=0 \\
& \frac{\partial \tau_{x y}}{\partial x}+\frac{\partial \sigma_{y}}{\partial y}+\frac{\partial \tau_{y z}}{\partial z}=0 \\
& \frac{\partial \tau_{x z}}{\partial x}+\frac{\partial \tau_{y z}}{\partial y}+\frac{\partial \sigma_{z}}{\partial z}=0
\end{aligned}
$$

where: $\sigma_{x}, \sigma_{y}, \sigma_{z}, \tau_{x y}, \tau_{x z}, \tau_{y z}$ are the components of the symmetrical stress tensor $\left(\tau_{x y}=\tau_{y x}\right.$, $\left.\tau_{x z}=\tau_{z x}, \tau_{y z}=\tau_{z y}\right)$ and $x, y, z$ - Cartesian coordinates.

Above equilibrium equations contain six unknowns and must be converted in order to be the functions of displacements $u_{x}, u_{y}, u_{z}$ because the number of equations must be the same as the number of unknowns. The components of stress tensor can be written in the following form: 


$$
\begin{gathered}
\sigma_{x}=\frac{E}{1+v}\left[\varepsilon_{x}+\frac{v}{1-2 v}\left(\varepsilon_{x}+\varepsilon_{y}+\varepsilon_{z}\right)\right] \\
\sigma_{y}=\frac{E}{1+v}\left[\varepsilon_{y}+\frac{v}{1-2 v}\left(\varepsilon_{x}+\varepsilon_{y}+\varepsilon_{z}\right)\right] \\
\sigma_{z}=\frac{E}{1+v}\left[\varepsilon_{z}+\frac{v}{1-2 v}\left(\varepsilon_{x}+\varepsilon_{y}+\varepsilon_{z}\right)\right] \\
\tau_{x y}=\frac{E}{2(1+v)} \gamma_{x y} \\
\tau_{y z}=\frac{E}{2(1+v)} \gamma_{y z} \\
\tau_{x z}=\frac{E}{2(1+v)} \gamma_{x z}
\end{gathered}
$$

where: $E$ - Young's modulus, $v$ - Poisson's ratio, $\varepsilon_{x}, \varepsilon_{y}, \varepsilon_{z}, \gamma_{x y}, \gamma_{x z}, \gamma_{y z}$ - the components of symmetrical strain tensor defined as follows:

$$
\begin{array}{ll}
\varepsilon_{x}=\frac{\partial u_{x}}{\partial x}, & \gamma_{x y}=\frac{\partial u_{x}}{\partial y}+\frac{\partial u_{y}}{\partial x} \\
\varepsilon_{y}=\frac{\partial u_{y}}{\partial y}, & \gamma_{y z}=\frac{\partial u_{y}}{\partial z}+\frac{\partial u_{z}}{\partial y} \\
\varepsilon_{z}=\frac{\partial u_{z}}{\partial z}, & \gamma_{x z}=\frac{\partial u_{x}}{\partial z}+\frac{\partial u_{z}}{\partial x}
\end{array}
$$

Equations (2), (3) make possible to convert equilibrium equations (1) into the functions of unknown displacements $u_{x}, u_{y}, u_{z}$ :

$$
\begin{aligned}
& f_{1} \frac{\partial^{2} u_{x}}{\partial x^{2}}+f_{3} \frac{\partial^{2} u_{x}}{\partial y^{2}}+f_{3} \frac{\partial^{2} u_{x}}{\partial z^{2}}+f_{2} \frac{\partial^{2} u_{y}}{\partial x \partial y}+f_{3} \frac{\partial^{2} u_{y}}{\partial y \partial x}+f_{2} \frac{\partial^{2} u_{z}}{\partial x \partial z}+f_{3} \frac{\partial^{2} u_{z}}{\partial z \partial x}=0 \\
& f_{2} \frac{\partial^{2} u_{x}}{\partial y \partial x}+f_{3} \frac{\partial^{2} u_{x}}{\partial x \partial y}+f_{3} \frac{\partial^{2} u_{y}}{\partial x^{2}}+f_{1} \frac{\partial^{2} u_{y}}{\partial y^{2}}+f_{3} \frac{\partial^{2} u_{y}}{\partial z^{2}}+f_{2} \frac{\partial^{2} u_{z}}{\partial y \partial z}+f_{3} \frac{\partial^{2} u_{z}}{\partial z \partial y}=0 \\
& f_{2} \frac{\partial^{2} u_{x}}{\partial z \partial x}+f_{3} \frac{\partial^{2} u_{x}}{\partial x \partial z}+f_{2} \frac{\partial^{2} u_{y}}{\partial z \partial y}+f_{3} \frac{\partial^{2} u_{y}}{\partial y \partial z}+f_{3} \frac{\partial^{2} u_{z}}{\partial x^{2}}+f_{3} \frac{\partial^{2} u_{z}}{\partial y^{2}}+f_{1} \frac{\partial^{2} u_{z}}{\partial z^{2}}=0
\end{aligned}
$$

where physical parameters $f_{1}, f_{2}, f_{3}$ are defined in the following way:

$$
\begin{gathered}
f_{1}=\frac{E(1-v)}{(1+v)(1-2 v)} \\
f_{2}=\frac{E v}{(1+v)(1-2 v)} \\
f_{3}=\frac{E}{2(1+v)}
\end{gathered}
$$

Presented mathematical model of elastic deformations is supplemented by the appropriate boundary conditions:

$$
\left.u_{x}\right|_{\Gamma} \neq 0,\left.\quad u_{y}\right|_{\Gamma}=\left.u_{z}\right|_{\Gamma}=0
$$




\section{Numerical model}

The numerical model is based on the finite element method [9] and is derived from the criterion of the method of weighted residuals [10]. Equations (4) are multiplied by the weighting function $w$ and integrated over the volume $\Omega$ of the deformable object. Weak form of equations (4) is shown below:

$$
\begin{gathered}
\int_{\Omega}\left(f_{1} \frac{\partial w}{\partial x} \frac{\partial u_{x}}{\partial x}+f_{3} \frac{\partial w}{\partial y} \frac{\partial u_{x}}{\partial y}+f_{3} \frac{\partial w}{\partial z} \frac{\partial u_{x}}{\partial z}\right) d \Omega+\int_{\Omega}\left(f_{2} \frac{\partial w}{\partial x} \frac{\partial u_{y}}{\partial y}+f_{3} \frac{\partial w}{\partial y} \frac{\partial u_{y}}{\partial x}\right) d \Omega+ \\
+\int_{\Omega}\left(f_{2} \frac{\partial w}{\partial x} \frac{\partial u_{z}}{\partial z}+f_{3} \frac{\partial w}{\partial z} \frac{\partial u_{z}}{\partial x}\right) d \Omega=\oint_{\Gamma} w p_{x} d \Gamma \\
\int_{\Omega}\left(f_{2} \frac{\partial w}{\partial y} \frac{\partial u_{x}}{\partial x}+f_{3} \frac{\partial w}{\partial x} \frac{\partial u_{x}}{\partial y}\right) d \Omega+\int_{\Omega}\left(f_{3} \frac{\partial w}{\partial x} \frac{\partial u_{y}}{\partial x}+f_{1} \frac{\partial w}{\partial y} \frac{\partial u_{y}}{\partial y}+f_{3} \frac{\partial w}{\partial z} \frac{\partial u_{y}}{\partial z}\right) d \Omega+ \\
+\int_{\Omega}\left(f_{2} \frac{\partial w}{\partial y} \frac{\partial u_{z}}{\partial z}+f_{3} \frac{\partial w}{\partial z} \frac{\partial u_{z}}{\partial y}\right) d \Omega=\oint_{\Gamma} w p_{y} d \Gamma \\
\int_{\Omega}\left(f_{2} \frac{\partial w}{\partial z} \frac{\partial u_{x}}{\partial x}+f_{3} \frac{\partial w}{\partial x} \frac{\partial u_{x}}{\partial z}\right) d \Omega+\int_{\Omega}\left(f_{2} \frac{\partial w}{\partial z} \frac{\partial u_{y}}{\partial y}+f_{3} \frac{\partial w}{\partial y} \frac{\partial u_{y}}{\partial z}\right) d \Omega+ \\
+\int_{\Omega}\left(f_{3} \frac{\partial w}{\partial x} \frac{\partial u_{z}}{\partial x}+f_{3} \frac{\partial w}{\partial y} \frac{\partial u_{z}}{\partial y}+f_{1} \frac{\partial w}{\partial z} \frac{\partial u_{z}}{\partial z}\right) d \Omega=\oint_{\Gamma} w p_{z} d \Gamma
\end{gathered}
$$

The weighting function $w$ depends on the method used in the process of spatial discretization. Presented model is based on the Galerkin method where $w$ is the same as the shape functions of the finite element used during discretization of considered volume. Final form of the global FEM equations is presented below:

$$
\mathbf{K} \cdot \mathbf{u}=\mathbf{B},
$$

where $\mathbf{K}$ is the global stiffness matrix, $\mathbf{B}$ - vector where known boundary displacements are stored, $\mathbf{u}$ - vector of unknown nodal displacements.

\subsection{Collision detection}

The collision occurs when the surface nodes of the moving object enter the interior of the stationary object (Fig. 2). Surface nodes of the source are then located within the boundary of finite elements of the target. The mesh may contain millions of elements, especially when it consists of tetrahedrons, so it is important to mark only those elements of the target that may collide with the source before starting of calculation process. Analogous selection also takes place in the elements of the source. This pre-selection of the surfaces of potential contact allows for huge savings in computational time during computer simulation. Collision is detected when any surface node of the source is inside the boundary of finite element of the target.

Fig. 3 shows the node $N$ that is inside the tetrahedral finite element $A B C D$ due to a collision. Collision detection is very simple in this case. At the beginning it is necessary to determine the volume $V$ of the tetrahedron $E$. Next, the volumes $V_{1}, V_{2}, V_{3}, V_{4}$ of 
tetrahedrons $A B C N, B D C N, A C D N, A B D N$ constructed from the successive walls of element $E$ and node $N$ are calculated.

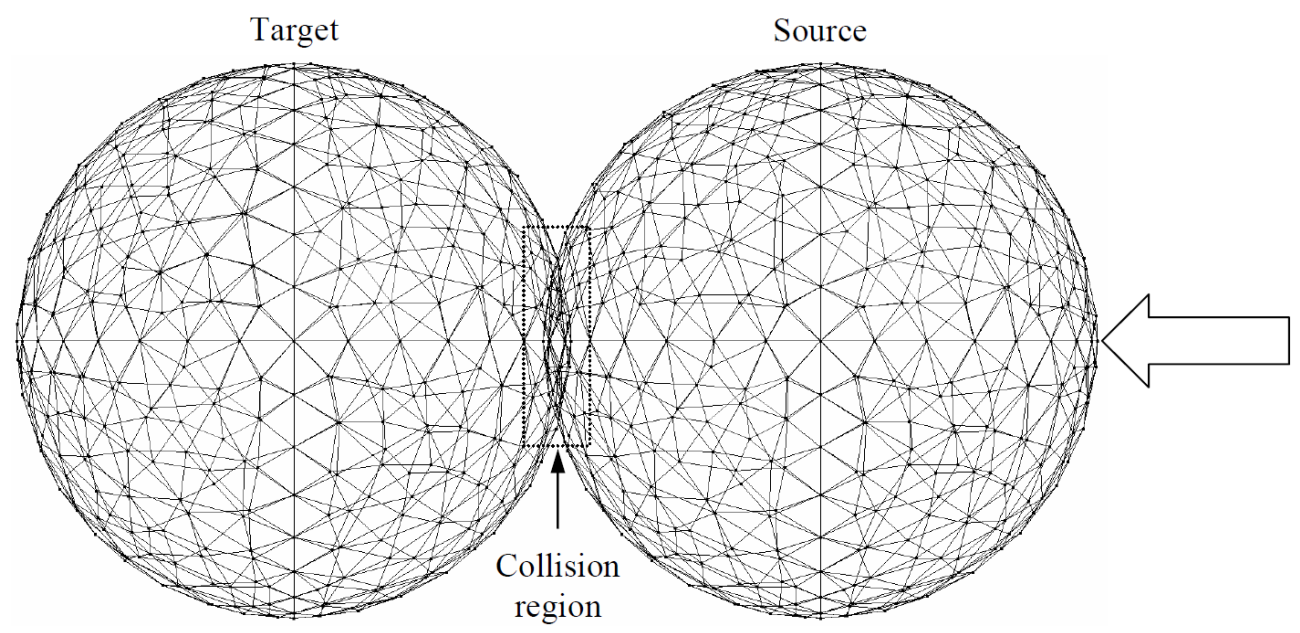

Fig. 2. Collision of objects

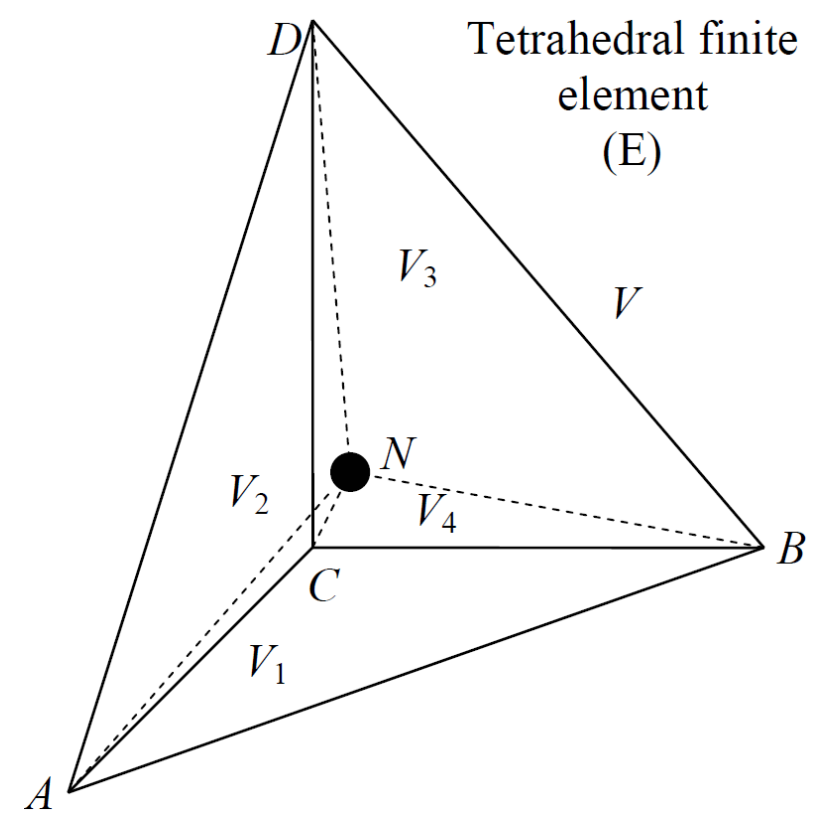

Fig. 3. A node inside a tetrahedral finite element during collision

If $N$ is inside $E$, the following condition must be fulfilled:

$$
V_{1}+V_{2}+V_{3}+V_{4}=V
$$

Equation (11) is the collision criterion. Each calculation step requires searching the set of contact nodes of the source and checking this criterion for all selected boundary elements of the target. Nodes that meet condition (11) are marked as "collision nodes" because they must be moved from the inside of the element to its surface. 


\subsection{Object deformation}

The surfaces of the objects in contact can not penetrate each other, so all the collision nodes must be translated to the surface of the target. The translation vector of each node is calculated perpendicular to the contact surface. In fact, the nodes are not directly moved, but the calculated translations modify vector $\mathbf{B}$. Then the displacements of each node are obtained after solving the set of equations (10). Modified shape of the collision region in the source is shown in Fig. 4.

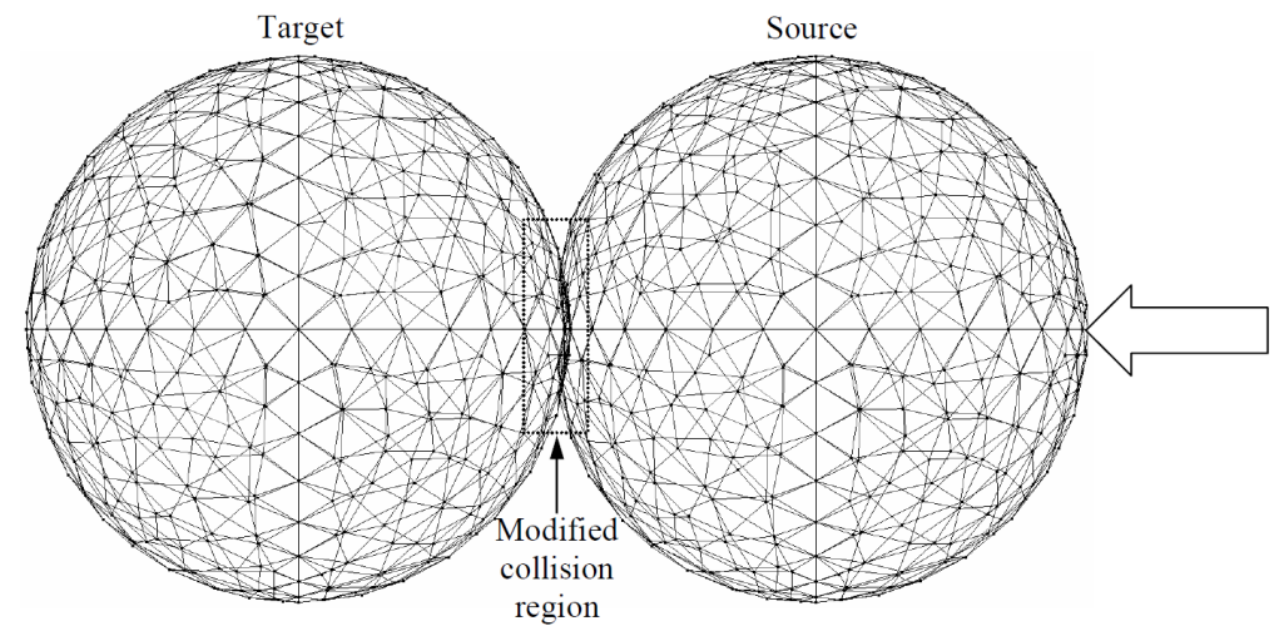

Fig. 4. Modification of the surface of the source after collision

The deformation of the source during contact progresses gradually step by step. It is important that the displacement of the source at each time step is less than the thickness of the surface layer of the elements in the mesh of the target. The algorithm during each time step can be expressed as follows:

1. Translation of the source

2. Detection of the collision nodes

3. Calculation of the boundary conditions in the collision region

4. Calculation of the nodal displacements of the source

5. Modification of the nodal coordinates

6. Return to point 1

\section{Example of calculation}

An original computer program using Visual $\mathrm{C}++2008$ has been written. Finite element meshes were prepared with the use of mesh generator GMSH 2.9. A system containing two balls of the same diameters $D=0.1 \mathrm{~m}$ is analyzed. The initial configuration of spatially discretized volumes is presented in Fig. 5. Each ball has an independent mesh built of tetrahedrons of average size $h_{e}=0.003 \mathrm{~m}$. First mesh contains 69747 elements with 12575 nodes, the second one has 67826 elements with 12251 nodes. Ball 1 is divided into finite elements despite the assumption of non-deformability because the algorithm for collision detection requires spatial discretization of each object in the system. Ball 2 is made of rubber with Young modulus $E=0.05 \mathrm{GPa}$ and Poisson's ratio $v=0.49$. Ball 2 moves along the $x$ axis at a speed $V_{x}=1 \mathrm{~mm} / \mathrm{s}$. Time step $\Delta t=1 \mathrm{~s}$, total time of the analysis $t_{\text {total }}=25 \mathrm{~s}$. On the right half of the surface of the ball 2, displacement boundary conditions are defined $u_{y}=u_{z}=0 \mathrm{~m}$. Displacement $u_{x}=1 \mathrm{~mm}$ is constant and cumulative due to modification of nodal 
coordinates at each step of analysis. This means that after 25 seconds the nodes on the right half of the ball 2 move along the $x$ axis by $25 \mathrm{~mm}$.

Ball 1

(non-deformable target of contact)

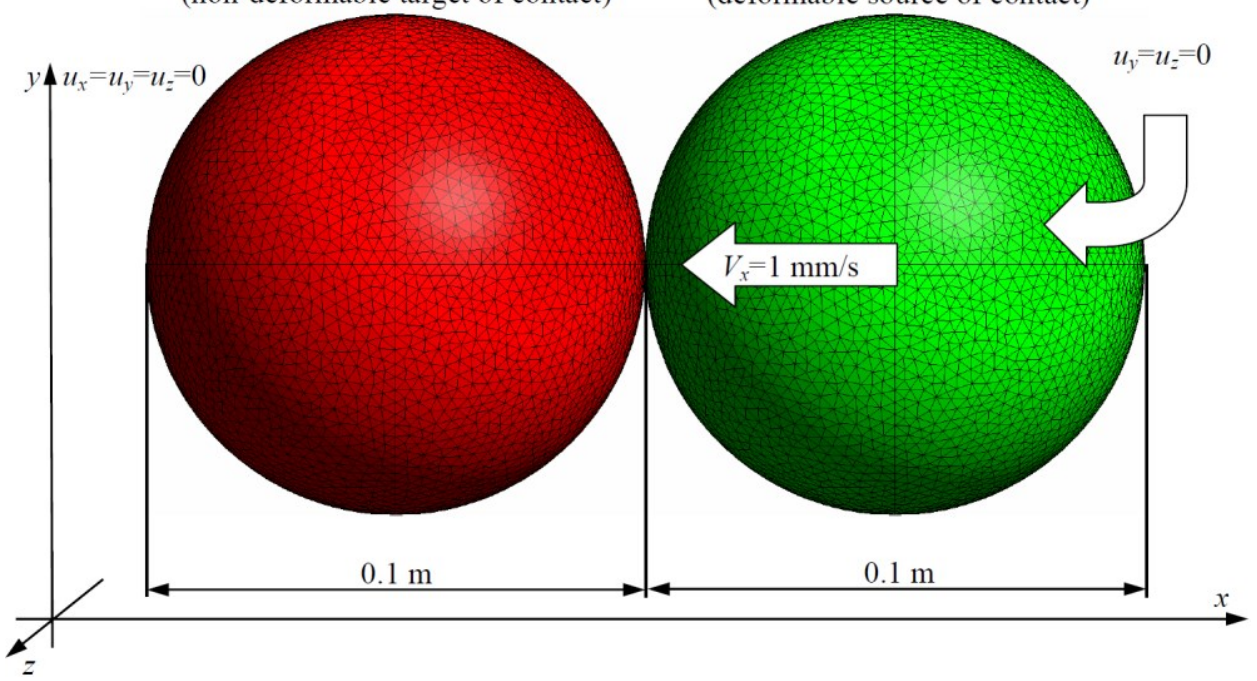

Fig. 5. Initial configuration of the system

The behaviour of balls in contact is presented in Fig. 6a-d. Right half of balls 2 remains unchanged due to assigned boundary conditions while the left one is subjected to large deformations.
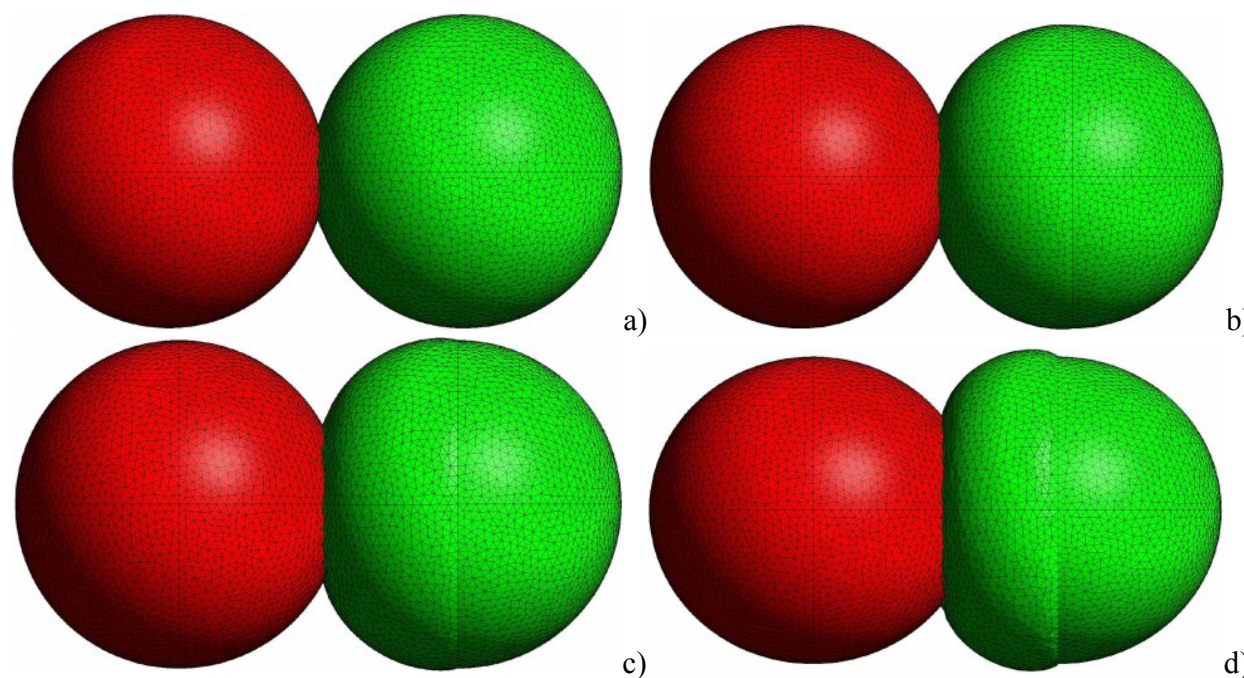

c)

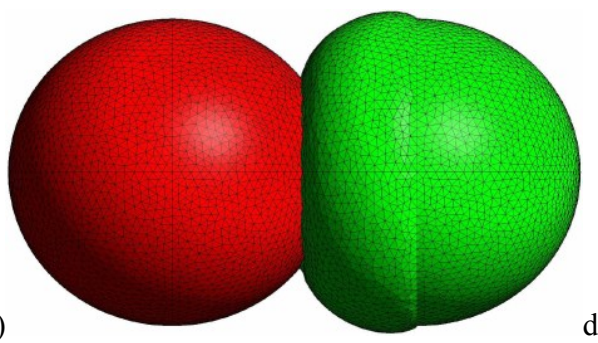

b)

Fig. 6. Four phases of contact a) $t=5 \mathrm{~s}, \mathrm{~b}) t=10 \mathrm{~s}$, c) $t=15 \mathrm{~s}, \mathrm{~d}) t=25 \mathrm{~s}$

Fig 7a-d show the stages of deformation of ball 2. It is clearly visible how the mesh of ball 2 fits the shape of ball 1 . The gradual increase in displacement allows for accurate modeling of even large deformations. 

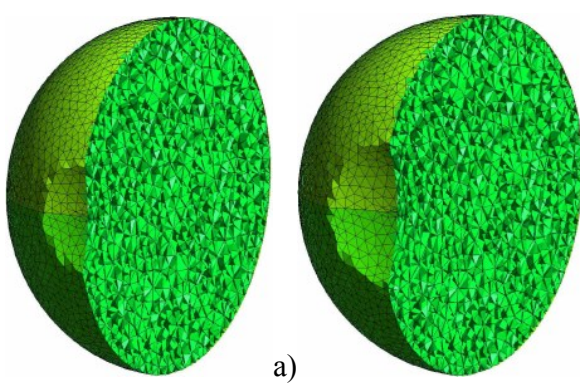

b)
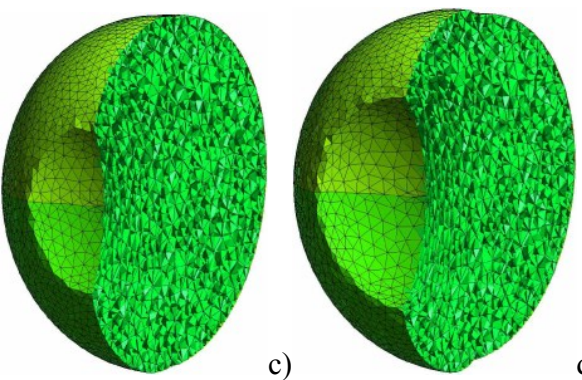

d)

Fig. 7. Cross section through ball 2 in the $x y$ plane during successive contact phases a) $t=5 \mathrm{~s}, \mathrm{~b}) t=10$ $\mathrm{s}, \mathrm{c}) t=15 \mathrm{~s}, \mathrm{~d}) t=25 \mathrm{~s}$

\section{Conclusions}

The numerical method of contact modeling in three dimensional space was presented. Described method of collision detection and modification of finite element meshes during the contact is straightforward and efficient. Presented approach may be useful in simulation of various processes occurring in the systems containing multiple elements. For example, the solidification of the metal in the mold is a process where two regions are in mechanical and also thermal contact. Further work will be focused on the expansion of the numerical model.

\section{References}

1. H. Hertz, Über die berührung fester elastischer Körper, Journal für die reine und angewandte Mathematik 92, 156-171 (1881)

2. K. L. Johnson, K. Kendall, A. D. Roberts, Surface energy and the contact of elastic solids, Proc. R. Soc. Lond. A. 324, 301-313 (1971)

3. B.V. Derjaguin, V.M. Muller, Y.P. Toporov, Effect of contact deformations on the adhesion of particles, J. Colloid Interface Sci. 53 (2), 314-326 (1975)

4. F.P. Bowden, D. Tabor, The area of contact between stationary and between moving surfaces, Proc. R. Soc. Lond. A. Mathematical and Physical Sciences 169 (938), 391413 (1939)

5. J.F. Archard, Elastic deformation and the laws of friction, Proc. R. Soc. Lond. A. Mathematical and Physical Sciences 243 (1233), 190-205 (1957)

6. L. Sowa, Z. Saternus, M. Kubiak, Numerical modelling of mechanical phenomena in the gantry crane beam Procedia Eng. 177, 225-232 (2017)

7. A. Bokota, T. Domański, Modelling and numerical analysis of hardening phenomena of tools steel elements, Arch Metall Mater. 54 (3), 575-587 (2009)

8. W. Piekarska, M. Kubiak, Z. Saternus, S. Stano, T. Domański, Numerical prediction of deformations in laser welded sheets made of X5CrNi18-10 steel, Arch Metall Mater. 60 (3), 1965-1972 (2015)

9. O.C. Zienkiewicz, Metoda elementów skończonych. (Arkady, Warszawa, 1972)

10. H. Grandin, Fundamentals of the Finite Element Method. (Waveland Press, Long Grove, 1991) 